\title{
TAURUS 2 Observations of Giant Extragalactic HII Regions
}

\author{
Héctor 0. Castañeda \\ Instituto de Astrofisica de Canarias, and Isaac Newton Group of \\ Telescopes, La Palma Observatory. E38200-La Laguna (Tenerife) Spain
}

\begin{abstract}
The use of TAURUS 2, a Fabry-Perot imaging spectrograph, for the study of the kinematics and the dynamics of giant extragalactic HII regions in nearby galaxies is discussed. The technical aspects of the instrument, together with the procedures adopted for data analysis and reduction, and some of the first scientific results of our programme are also presented.
\end{abstract}

\section{Introduction}

The study of the kinematics and dynamics of the ionized gas of giant extragalactic HII regions (GEHR) may expand our understanding of the mechanism which triggers star formation, and about the possible processes that influence their chemical evolution. Imaging spectroscopy is an ideal observational tool, combining high spectral resolution with "multiplexing" spatial abilities that permit the coverage of a complete object. Together with C. Muñoz-Tuñon, J.M. Vilchez, O. Fuentes, and N. Sabalisck (IAC), R. Terlevich (RGO) and M. Copetti (U. Santa Maria) a long term programme is being conducted to study the kinematics of the gas in the main complexes of nearby galaxies, using the TAURUS 2 Fabry Perot Imaging Spectrograph at the Observatorio del Roque de los Muchachos, with the aim of studying the spatial distribution and the velocity structure of the ionized gas and understanding the interactions between the different gas components (neutral, ionized, and molecular) of the objects. The first part of the article describes the technical aspects of Fabry-Perot spectroscopy with TAURUS 2, including a description of the instrument and the process of acquiring, reducing, and analysing the data. The second part discusses some of the physical results obtained during the programme.

\section{The instrument}

The TAURUS-2 Fabry Perot imaging spectrograph was built to operate as a wide-field imaging Fabry-Perot interferometer and for two-dimensional imaging. The instrument is located in the $\mathrm{f} / 11$ Cassegrain focus of the WHT, and by means of an optical array the focal ratio of operation can be set at $f / 2$ or $f / 4$. It is designed to obtain spectra over a field of up to $9 \mathrm{arcmin}$. The etalon wheel has 6 positions, of which 4 can be used for FP etalons. The etalons are maintained in parallelism by means of a Queensgate Instruments CS-100 servo-stabilized 
system. A set of narrow band filters of a suitable wavelength redshift, matching the free spectral range of the spectrograph are available. Two types of detectors can be used: an image photon counting system, known as IPCS-II, and several CCDs.

\section{Observations}

For this observational programme two-dimensional imaging spectroscopy in the $\mathrm{H} \alpha$ and $[\mathrm{OIII}] \lambda 5007$ lines was obtained for the main regions of $\mathrm{M} 101, \mathrm{M} 33$, NGC 2403, NGC 2366 and NGC 6822, during two runs in July 1990 and January 1991. The 125 microns gap etalon was used, each data cube of 100 frames of $256 x$ 256 pixels, for a total integration time of $3600 \mathrm{sec}$. The pixel size was 0.26 ". The IPCS-II served as detector; it has a lower quantum efficiency than a CCD, but allows to scan through a data cube very rapidly, averaging possible variations in atmospheric transparency $A$ top flat interference filter at the appropriate redshift of each object was used to isolate a single order of interference. A calibration cube was obtained at the beginning of the night, observing a nearby emission line of the CuNe lamp., which was later used to obtain the free spectral range and to compute the etalon gap. To monitor any mechanical deformations of the etalon, and to check its optical stability a calibration ring was obtained at the beginning and end of each observation.

\section{Data Analysis and Reduction}

The raw data was phase corrected using the TAUCAL calibration software package (Lewis and Unger 1992). The final product of an observation was a calibrated data cube, with two axes in spatial coordinates, and the third the wavelength dimension. Special features of the reduction software included the possibility of correcting the spatial distorsion caused by the IPCS, as well as a programme (TAUFITS) that performs interactive or automatic fitting analyses of the data cube. Given a data cube, the programme can automatically fit either gaussians or Cauchy functions, with different bin sizes, and selecting particular areas of analysis. The fitting criteria established as "true" points those which showed a larger line width in the fit than the instrumental line width. Four main param. eters can be obtained from the fit: line intensity, continuum intensity, velocity and line width.

\section{Discussion}

Gaseous nebulae have Reynolds numbers of $\approx 10^{6}$, so their mass flow is likely to occur in a chaotic, random state. Typical values for the velocity dispersion of GEHR along the line of sight $(\sigma)$ quoted in the literature are $9-22 \mathrm{~km} \mathrm{~s}^{-1}$, hence highly supersonic. The intrinsic broadening of a spectra (after instrumental, quantum, and thermal width corrections) -which we term "turbulence"could occur as a result of virialized motions, stellar winds, or also as a manifestation of other physical processes such as collapse of the gas, a large scale gradient of the radial velocity or geometric effects (Castañeda 1988). For ex- 
ample, while synthetic aperture spectra, with large aperture, in general show gaussian line profiles, spectra obtained with small apertures reveal asymmetric line profiles and multiple components. The study of the velocity maps of the regions uncovers systematic motions in the form of velocity gradients with a well-defined pattern, confirmed by comparison of velocity maps in different emission lines. The velocity dispersion depends on the selected aperture, and extreme care should be taken before assigning a single value to the region.

An example is NGC 588 (GEHR in M 33), whose morphology is very similar to a planetary nebula's: it has an elliptical shape, with dimensions of 21.3 arc sec (major axis) by 12 arcsec (minor axis). An analysis of the velocity field was carried out using the TAUFITS program, with a bin size of 1.8 arcsec (Castañeda et al. 1992). The velocity histogram in $\mathrm{H} \alpha$ is clearly asymmetric; taking as reference the heliocentric velocity of the object, the velocity ranges from $-7 \mathrm{~km}$ $\mathrm{s}^{-1}$ (blueshifted) to $+23 \mathrm{~km} \mathrm{~s}^{-1}$ (redshifted). The same behaviour is observed in [OIII]. The more blueshifted velocities are associated with the central part of the region. This is also the area where the dispersion along the line of sight is larger. The velocity dispersion $(\sigma)$ histogram for both emission lines is similar: the turbulent component ranges between $5-30 \mathrm{~km} \mathrm{~s}^{-1}$. If we take the sound velocity in one dimension as $\approx 8 \mathrm{~km} \mathrm{~s}^{-1}$, we see that most of the gas is in a state of supersonic turbulence. The power of imaging spectroscopy is beautifully illustrated in the case of NGC 2363, a giant HII region in the Magellanic irregular galaxy NGC 2366, at a distance of 3.5 Mpc. Roy et al. (1991) found line splitting in the [OIII] line, which they associated to a bubble of $200 \mathrm{pc}$ in diameter, at a velocity expansion of $45 \mathrm{~km} \mathrm{~s}^{-1}$. The object was observed by us in $\mathrm{H} \alpha$ and [OIII]. The line in [OIII] is split, with a separation between the two components of 81 $\mathrm{km} \mathrm{s}^{-1}$ comparable with the values of the literature, but no line splitting was detected in the $H \alpha$ profiles. Skillman and Balick (1984) also detected different kinematical behaviour in their high resolution echelle data in both emission lines, which they suggested was due to high temperature gas within the nucleus of the region. In order to visualize the expanding structure, Fig. 1 shows the collapsed image of the data cube in [OIII], as well as a partial collapse ( 2 planes $=15 \mathrm{~km} \mathrm{~s}^{1}$ ) centered on the plane 53, where the global [OIII] line has its center in heliocentric velocity. The shell is asymmetric ( 6.4 arcsec $\mathrm{E}-\mathrm{W}, 5.9$ arcsec $\mathrm{N}-\mathrm{W}$ ), but its dimensions are smaller from those reported by Roy et al.. Two possibilities could explain the discrepancy: i) the lower integration time (3600 sec vs. $30000 \mathrm{sec}$ ), and the smaller resolution power of our data, which does not allow the line splitting in the periphery to be observed.

The expanding structure could be due to stellar winds. The stellar population is not known, but a recent paper of Drissen et al. (1993) presenting narrow band imaging suggests that about 5 Wolf-Rayet stars are located near the secondary eastern knot of NGC 2363 (note that a nebular origin of the emission cannot be excluded). We have established that the WR excess is located in position $(120 ; 120)$ in Fig. 1, clearly off-center with respect to the center of symmetry of the bubble.

Acknowledgments. I gratefully acknowledge the Spanish Directorate General for Scientific adn Technical Research (DGICYT) for a fellowship under which part of this work was carried out, and Monica Murphy for her kindness while correcting the English text. The WHT is operated on the island of La 

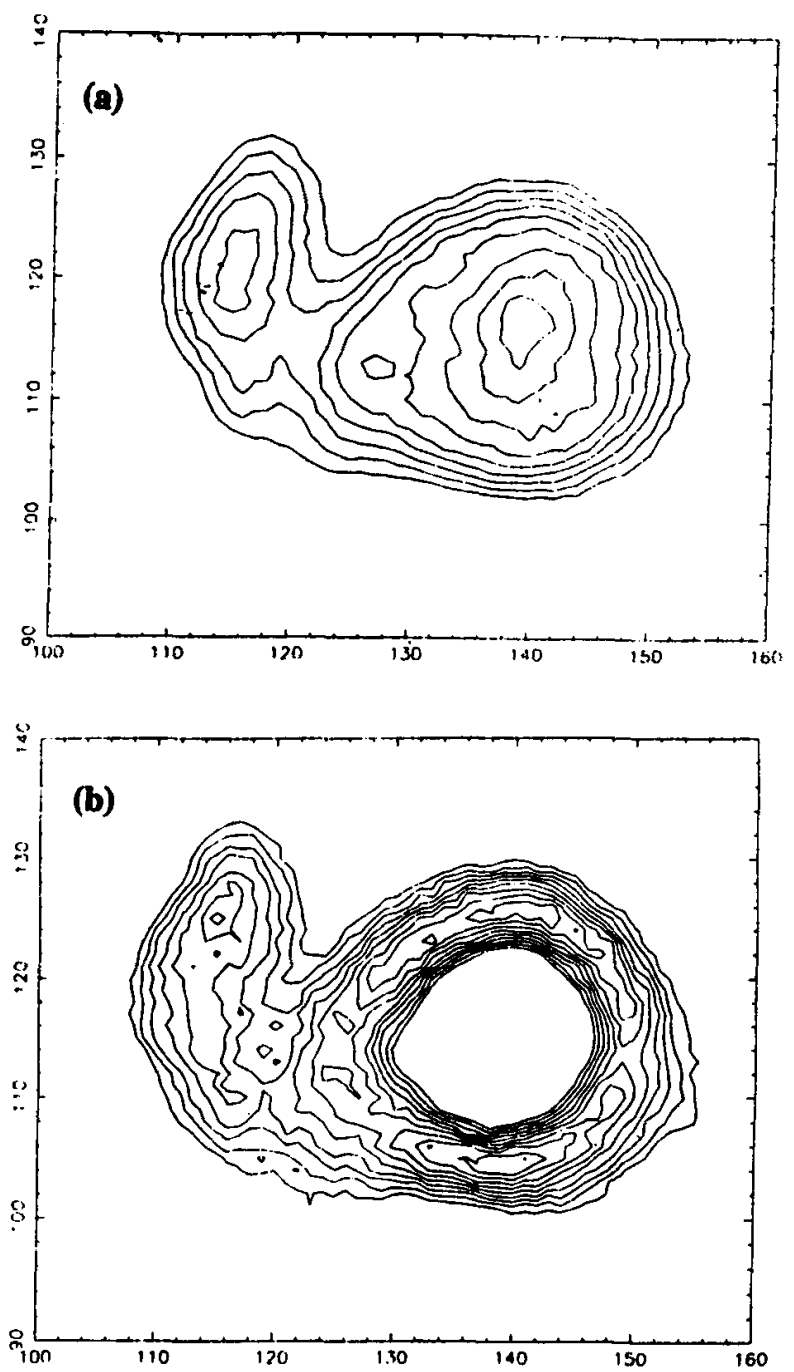

Figure 1. Figure 1: (a) [OIII] $\lambda 5007$ image from the collapse of the data cube in the spectral direction; (b) collapse of the planes $(53,54)$, equivalent to a velocity interval of $15 \mathrm{~km} \mathrm{~s}^{-1}$ :: the shape of the bubble is clearly visible. Both images show 10 isocontours, from maximum count on the image to $40 \%$ of the peak. North is up, East to the left; 1 pixel $=0.26$ arcsec. 
Palma by the Royal Greenwich Observatory at the Spanish Observatorio del Roque de los Muchachos of the Instituto de Astrofísica de Canarias.

\section{Discussion}

Dr. Bland-Hawthorn: Your comparison of the [OIII] and $\mathrm{H} \alpha$ distribution is very interesting. The rapid change in the [OIII] at the line core may arise from saturation of the IPCS. Have you ruled out this possibility?

Dr. Castañeda: The dip of the emission line below the continuum level might be explained by underlying broad emission, which it is in fact observed in the region (Roy et al. 1992). Agreement between the line splitting value and in the shape of the shell suggests that the feature is real, and not a saturation effect. Nevertheless, I agree with you that the only way to settle this issue is by repeating the observations using a CCD detector.

Dr. Bland-Hawthorn: How do you separate bulk motions from turbulent motions in your data?

Dr. Castañeda: At present, several strategies are being developed. Individual fitting of the spectra is being carried out in some regions, to study the different components of the velocity field. Automatic measurement of the skewness of the line profile may also give an idea about mass motions in the regions. Finally, the use of statistical technics such as the structure function (after removal of systematic motions in the gas) will provide information about the "turbulent" nature of the flow (in the Kolmogorov sense).

\section{References}

Castañeda, H.O. 1988, ApJS, 67, 93.

Castañeda, H.O., Copetti, M.V.F., Muñoz-Tuñon, C. 1992, BAAS, 24, 816.

Drissen, L., Roy, J.-R., \& Moffat, A.F.J. 1993, AJ, 106, 1460.

Lewis, J.R. \& Unger, S.W. 1992, in Astronomical Data Analisis and Software I, eds. D.M. Worrall, C. Biesmesderfer, J. Barnes, Astronomical Society of the Pacific Ser. Vol. 25, p. 445.

Roy, J.-R., Boulesteix, J., Joncas, G. \& Grundseth, B. 1991, ApJ, 367, 141.

Roy, J.-R., Aubé, M., McCall, M.L., \& Dufour, R.J. 1992, ApJ, 386, 498.

Skillman, E.D., Balick, B. 1984, ApJ, 280, 580. 